\title{
Direct determination of quasar redshifts
}

\author{
J. H. J. de Bruijne ${ }^{1}$, A. P. Reynolds ${ }^{1}$, M. A. C. Perryman ${ }^{1,2}$, A. Peacock ${ }^{1}$, F. Favata ${ }^{1}$, N. Rando ${ }^{1}$, \\ D. Martin ${ }^{1}$, P. Verhoeve ${ }^{1}$, and N. Christlieb ${ }^{3}$ \\ 1 Astrophysics Division, European Space Agency, ESTEC, Postbus 299, 2200AG Noordwijk, The Netherlands \\ 2 Sterrewacht Leiden, Postbus 9513, 2300RA Leiden, The Netherlands \\ 3 Hamburger Sternwarte, Gojenbergsweg 112, 21029 Hamburg, Germany
}

Received 2 November 2001 / Accepted 26 November 2001

\begin{abstract}
We present observations of 11 quasars, selected in the range $z \approx 2.2-4.1$, obtained with ESA's Superconducting Tunnel Junction (STJ) camera on the WHT. Using a single template QSO spectrum, we show that we can determine the redshifts of these objects to about $1 \%$. A follow-up spectroscopic observation of one QSO for which our best-fit redshift $(z=2.976)$ differs significantly from the tentative literature value $(z \approx 2.30)$ confirms that the latter was incorrect.
\end{abstract}

Key words. instrumentation: detectors - galaxies: distances and redshifts - galaxies: high-redshift quasars: absorption lines - quasars: emission lines - quasars: general

\section{Introduction}

Large ground and space telescopes combined with solid state detectors have revolutionized optical astronomy over the past two decades, yet deriving physical diagnostics of stars and galaxies still requires the somewhat indirect methods of filter photometry or dispersive spectroscopy to measure spectral features, energy distributions, and redshifts. The recent development of high-efficiency superconducting detectors (Perryman et al. 1993; Peacock et al. 1996) has introduced the possibility of measuring individual optical photon energies directly, and the first high time-resolution spectrally-resolved observations of rapidly variable sources such as cataclysmic variables and optical pulsars using these techniques have been reported (Perryman et al. 1999; Romani et al. 1999; Perryman et al. 2001; Bridge et al. 2001). Many extensive observational programmes which aim at determining the largescale structure of the Universe, and galaxy formation and evolution (e.g., the Sloan Digital Sky Survey, Fan et al. 1999; the Anglo-Australian Telescope 2dF survey, Croom et al. 2001), demand high-efficiency extragalactic spectroscopy. Here we report the first optical measurements of spectral energy distributions of quasars using an imaging detector with intrinsic energy resolution, and show that we can determine their redshifts directly with excellent precision.

Send offprint requests to: J. H. J. de Bruijne, e-mail: Jos.de.Bruijne@esa.int

\section{Observations}

We observed 11 quasars in the redshift range $z=$ $2.2-4.1$, the sample comprising relatively bright highredshift Lyman-limit quasars from the published literature (Sargent et al. 1989), supplemented by three lower redshift objects, two of which were discovered in objective prism-type surveys (Table 1). Observations used the ESA superconducting tunnel junction (STJ) camera, SCam2 (Rando et al. 2000), on the 4.2-m William Herschel Telescope, La Palma, between 2000 October 1-4. The camera is a $6 \times 6$ array of $25 \times 25 \mu \mathrm{m}^{2}\left(0.6 \times 0.6 \operatorname{arcsec}^{2}\right)$ tantalum junctions, providing individual photon arrival time accuracies to about $5 \mu \mathrm{s}$, a resolving power of $\mathcal{R} \approx 8$ at $\lambda=500 \mathrm{~nm}$, and high sensitivity from $310 \mathrm{~nm}$ (the atmospheric cutoff) to about $720 \mathrm{~nm}$ (currently set by long-wavelength filters to reduce the thermal noise photons). All objects show strong Ly- $\alpha$ and CIV emission lines which, at these redshifts, will be present within our wavelength response range. Observations were made in modest seeing (1-1.5 arcsec at airmass $X=1)$, and at air-masses between $X=1.07-1.82$.

\section{Data reduction}

Information on each detected photon consists of arrival time, $x, y$ coordinates of the junction, and an energy channel in the range 0-255. A photon of energy $E_{\mathrm{p}}$ (in eV) incident on a particular junction is assigned to an energy channel $E_{i}=G \cdot E_{\mathrm{p}}+C$, where each pixel is characterised 
Table 1. The 11 quasars observed. $V$ gives the catalogue magnitude (probably questionable for 2143-158). $T$ gives the exposure time in seconds, and $z_{\text {obs }}$ our estimated redshift. The final two columns give the literature redshift and its source: S89 = Sargent et al. (1989) [spectroscopy]; M77 = MacAlpine et al. (1977) [objective prism]; C91 = Chaffee et al. (1991) [spectroscopy]; C85 = Crampton et al. (1985) [grens plate].

\begin{tabular}{rccrccc}
\hline \hline Obs. & $\begin{array}{c}\text { QSO } \\
\text { name }\end{array}$ & $\begin{array}{c}V \\
(\mathrm{mag})\end{array}$ & $\begin{array}{c}T \\
(\mathrm{~s})\end{array}$ & $z_{\text {obs }}$ & $z_{\text {lit }}$ & $\begin{array}{c}\text { Lit. } \\
z\end{array}$ \\
\hline 1 & $0000-263$ & 17.5 & 600 & 4.095 & 4.111 & $\mathrm{~S} 89$ \\
2 & $0052-009$ & 18.2 & 1033 & 2.190 & 2.212 & $\mathrm{C} 91$ \\
3 & $0055-264$ & 17.5 & 600 & 3.625 & 3.656 & $\mathrm{~S} 89$ \\
4 & $0127+059$ & 18.0 & 600 & 2.976 & 2.30 & $\mathrm{M} 77$ \\
5 & $0132-198$ & 18.0 & 900 & 3.073 & 3.130 & $\mathrm{~S} 89$ \\
6 & $0148-097$ & 18.4 & 1800 & 2.845 & 2.848 & $\mathrm{~S} 89$ \\
7 & $0153+045$ & 18.8 & 600 & 2.978 & 2.991 & $\mathrm{~S} 89$ \\
8 & $0302-003$ & 18.4 & 900 & 3.263 & 3.286 & $\mathrm{~S} 89$ \\
9 & $0642+449$ & 18.5 & 900 & 3.366 & 3.406 & $\mathrm{~S} 89$ \\
10 & $2143-158$ & 21.2 & 1800 & 2.296 & 2.3 & $\mathrm{C} 85$ \\
11 & $2233+136$ & 18.6 & 900 & 3.110 & 3.209 & $\mathrm{~S} 89$ \\
\hline \hline
\end{tabular}

by its own gain $G$ (in channels per eV) and offset $C$ (in channels). Laboratory measurements have confirmed that all 36 junctions have a highly linear, albeit slightly pixeldependent, energy response. Calibration consists of first bringing the observed energy channels to a common reference scale, corresponding to an arbitrary reference pixel, using a fixed gain map based on laboratory measurements. The offset of the reference pixel is constant $(C=-2.0)$, and its gain is then the only free parameter in the absolute energy calibration. Small temporal gain variations resulting from bias voltage drifts and small detector temperature variations $( \pm 0.01 \mathrm{~K}$ on the nominal operating temperature of $\approx 0.32 \mathrm{~K}$ ) are monitored and calibrated.

Subtraction of the appropriate sky contribution for each quasar spectrum can in principle be based on the background signal in the outer array junctions, but given the small array size and seeing and refraction effects, we generally also took a nearby sky frame immediately following each quasar observation. Most observations were taken in astronomically dark time; QSO 2233+136, 2143-158, and 0148-097 were observed with the Moon setting, with background subtraction slightly less accurate.

\section{Results}

We have determined each quasar redshift by comparing the calibrated energy distributions, $f_{\text {obs }}\left(E_{i}\right)$, with a single rest-frame composite quasar spectrum (Zheng et al. 1997) based on 284 Hubble Space Telescope Faint Object Spectrograph spectra of 101 quasars with $z>0.33$. For a given gain $G$ and redshift $z$, we construct the model energy-channel distribution $f_{\bmod }\left(E_{i}\right)$, as follows. The template spectrum is shifted from the rest frame to redshift $z$, and a mean accumulated absorption of the Lyman forest for this redshift is introduced (Møller \& Jakobsen 1990) (all our objects are at high Galactic latitude, and we neglect Galactic reddening). The resulting spectrum is

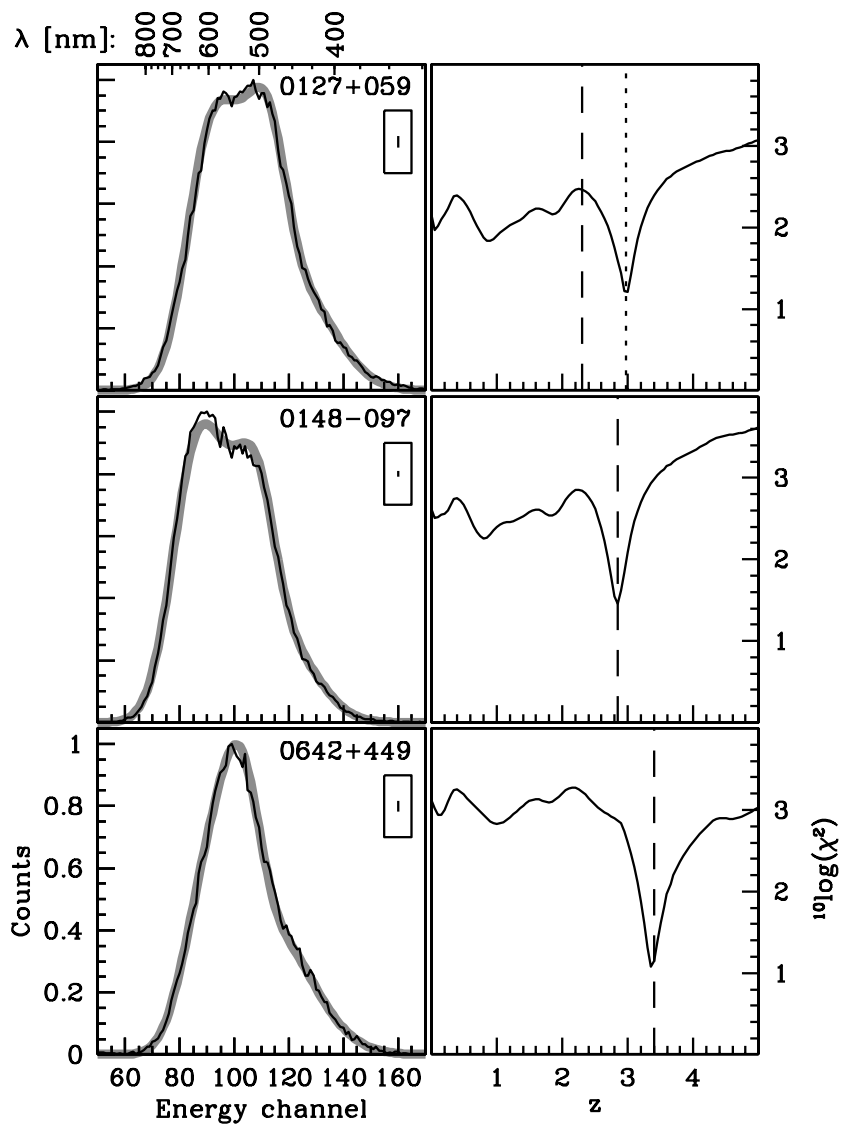

Fig. 1. Results for QSO 0127+059, 0148-097, and 0642+449. Left: the observed (black curves) and modeled (grey curves) energy channel distributions (arbitrary units). Insets indicate the estimated Poisson noise. Numbers above the top left panel show the mapping between energy channel and wavelength. Right: the corresponding dependence of $\chi^{2}$ on $z$. Vertical dashed lines indicate the literature redshifts; the dotted line for QSO 0127+059 indicates the spectroscopic redshift reported in this letter $(z=3.04)$.

corrected for the mean atmospheric transmission at the relevant airmass, adjusted for the instrument and telescope efficiency curves and exposure time, transformed from wavelength spectra to energy-channel spectra, and finally convolved with a suitable Gaussian in order to account for the finite energy resolution of the detector. We then derive redshift $z$ and gain $G$ (and a normalization constant $N$ ), by minimizing the classical $\chi^{2}$ function:

$\chi^{2}(z, G, N)=\frac{1}{118} \cdot \sum_{i=50}^{170}\left[\frac{f_{\mathrm{obs}}\left(E_{i}\right)-N \cdot f_{\bmod }\left(E_{i}\right)}{\sigma_{f_{\mathrm{obs}}}\left(E_{i}\right)}\right]^{2}$

using a downhill simplex routine (Press et al. 1995). Summation extends over relevant energy channels, and 118 is the number of degrees of freedom. Resulting gains are in the range $42-45$ channels $\mathrm{eV}^{-1}$, consistent with laboratory calibration. The resulting redshifts are listed in Table 1, along with the literature values. Examples of the observed and modeled spectra are shown in Fig. 1. The overall shape of these spectra, and in particular the sharp falloff at low energy channels, is due to the response of 


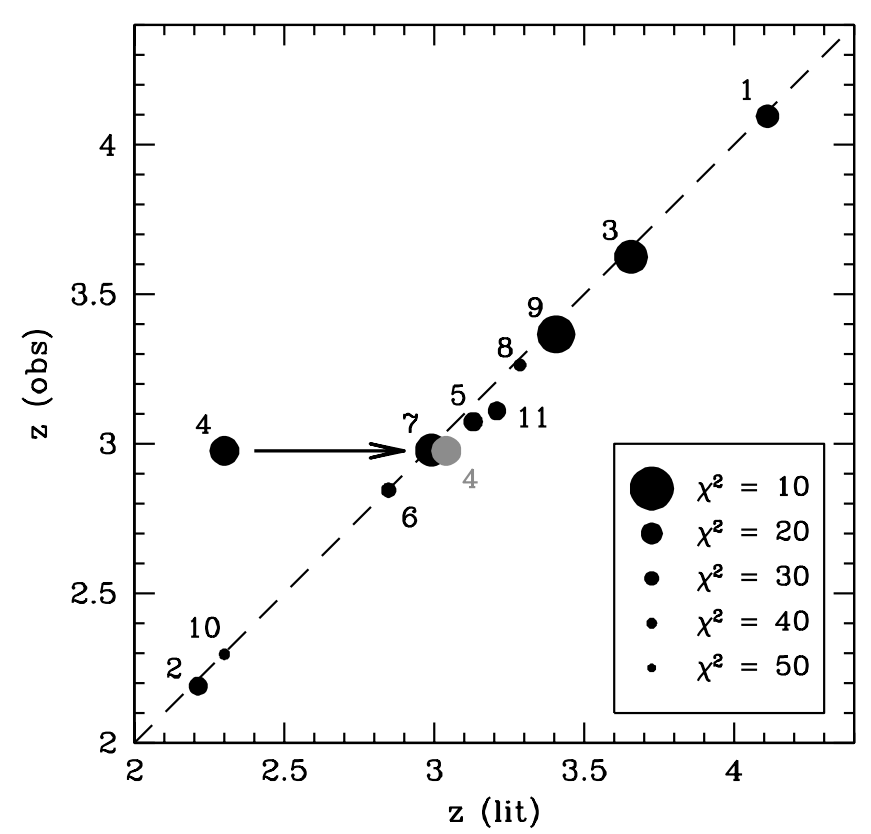

Fig. 2. Observed versus literature redshifts. Numbers refer to the objects listed in Table 1, and symbol sizes correspond to $\chi^{2}$ (smaller symbols indicating a poorer fit). QSO $0127+059$ has an incorrect literature redshift of 2.30; our spectroscopic followup observation yields $z=3.04$, moving the point to the position shown in grey. The dashed line shows the nominal 1:1 correlation.

the instrument and telescope. In practice, the Ly- $\alpha$ emission line and the associated break at shorter wavelengths contribute most to the redshift determination. Figure 2 compares the best-fit redshifts with the literature values.

QSO $0127+059$ is our single prominent outlier. It was discovered in a thin prism survey (MacAlpine et al. 1977), classified as a possible quasar, and tentatively assigned a redshift of $z \approx 2.30$, but with an uncertain line identification. Although the quality of our fit is acceptable (Fig. 1), our derived redshift, $z=2.976$, differs significantly from the literature value. We subsequently obtained a $1200 \mathrm{~s}$ spectrum of QSO $0127+059$ (Fig. 3) with the Siding Spring Observatory 2.3-m telescope. The wavelength coverage (not optimised for quasar spectroscopy) was $345-537$ and $560-753 \mathrm{~nm}$, using the Double Beam Spectrograph with dichroic 1 and gratings 600B and 600R. We determine a spectroscopic redshift $z=3.04$, which agrees with our estimate to about $2 \%$ (Fig. 2).

A small systematic offset in the overall correlation, of $\approx 0.03$ in $z$, can be attributed to a small mismatch in the shape of the energy broadening function or the overall throughput used in the modeling. The mean scatter for all observations is $\sigma_{z}=0.03$; removing the systematic offset, 8 of the 11 objects agree to within $1 \%$. Several factors, such as gain variations, erroneous sky subtraction or extinction correction (e.g., due to unmodeled seasonal Saharan dust in the atmosphere), or template mismatch at the object level (related to continuum slope, line ratios, etc.), may explain the observed spread. Formally, none of the fits is particularly good, in the sense that none of them

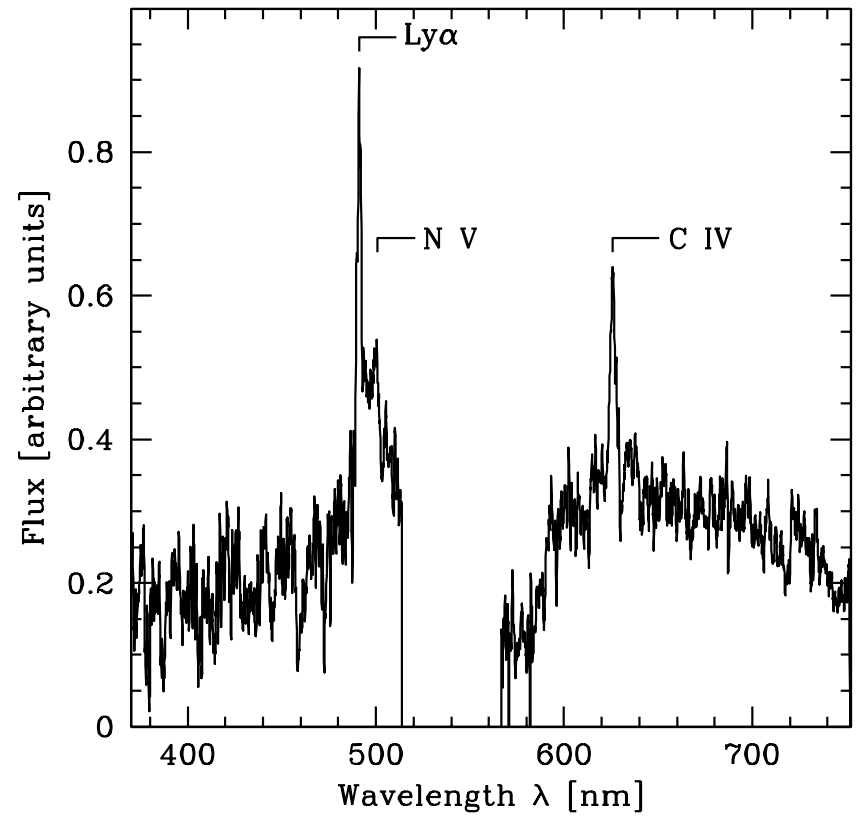

Fig. 3. The spectrum of QSO $0127+059$ obtained with the Siding Spring Observatory 2.3-m telescope, and smoothed with a $15 \AA F W H M$ Gaussian. We determine $z=3.04$; the resulting redshifted locations of Ly- $\alpha(121.6 \mathrm{~nm}), \mathrm{NV}(124.0 \mathrm{~nm})$, and CIV $(154.9 \mathrm{~nm})$ are indicated.

has reduced $\chi^{2} \approx 1$. A key factor in $\chi^{2}$-statistics, however, is the absence of systematic errors, which will exist here in part due to template mismatch, although details are largely hidden as a result of the limited detector resolution. The general consistency between the models and the observations, and the pronounced, deep and narrow, minima in all $\chi^{2}$ versus $z$ plots, nonetheless indicate that our fits, as a set, are acceptable.

The pronounced minima are apparent in our data sets truncated a posteriori to observation times as small as, e.g. 10-20 s for the $z=4.1$ object QSO 0000-263, where $\approx 350$ source photons $\mathrm{s}^{-1}$ were recorded.

\section{Discussion}

Although extraction of detailed physical information from the spectra is limited by the modest resolving power $(\mathcal{R} \approx 8)$ of the current device, a significant improvement in energy resolution can be expected in the future (Perryman et al. 1993; Peacock et al. 1997), and additional template spectra could then be used for model fitting. Our results show that low-resolution spectroscopy of faint extragalactic sources is possible with these devices, enabling the determination of redshift, and perhaps morphological type and emission and absorption line ratios (Jakobsen 1999; Mazin \& Brunner 2000). Our instrument development is aimed at larger format arrays to facilitate sky subtraction and possibly for multi-object spectroscopy, and an increased energy resolution to improve physical diagnostic capability. An overall wavelength response extending further to the red, consistent with the fundamental 
device response characteristics, would also open up a larger accessible redshift range.

Acknowledgements. The William Herschel Telescope is operated on the island of La Palma by the Isaac Newton Group (ING) in the Spanish Observatorio del Roque de los Muchachos of the Instituto de Astrofísica de Canarias. We thank J. Verveer and S. Andersson for instrument contributions, P. Jakobsen for advice on the template spectrum, I. Busa and B. Fuhrmeister for obtaining and reducing the spectrum of QSO 0127+059, and the referee, Scott Croom, for helpful comments. This research has made use of the ADS (NASA) and SIMBAD (CDS) services.

\section{References}

Bridge, C. M., Cropper, M., Ramsay, G., et al. 2001, MNRAS, submitted

Chaffee, F. H., Foltz, C. B., Hewett, P. C., et al. 1991, AJ, 102, 461

Crampton, D., Schade, D., \& Cowley, A. P. 1985, AJ, 90, 987 Croom, S. M., Smith, R. J., Boyle, B. J., et al. 2001, MNRAS, 322, L29

Fan, X., Strauss, M. A., Schneider, D. P., et al. 1999, AJ, 118, 1

Jakobsen, P. 1999, in Ultraviolet-Optical Space Astronomy Beyond HST, ASP Conf. Ser., 164, 397
MacAlpine, G. M., Smith, S. B., \& Lewis, D. W. 1977, ApJS, 35, 197

Mazin, B. A., \& Brunner, R. J. 2000, AJ, 120, 2721

Møller, P., \& Jakobsen, P. 1990, A\&A, 228, 299

Peacock, A., Verhoeve, P., Rando, N., et al. 1996, Nature, 381, 135

Peacock, T., Verhoeve, P., Rando, N., et al. 1997, A\&AS, 123, 581

Perryman, M. A. C., Cropper, M., Ramsay, G., et al. 2001, MNRAS, 324, 899

Perryman, M. A. C., Favata, F., Peacock, A., Rando, N., \& Taylor, B. G. 1999, A\&A, 346, L30

Perryman, M. A. C., Foden, C. L., \& Peacock, A. 1993, Nuc. Inst. Meth. A, 325, 319

Press, W. H., Teukolsky, S. A., Vetterling, W. T., \& Flannery, B. P. 1995, Numerical Recipes in Fortran; the Art of Scientific Computing, 2nd edition (Cambridge University Press)

Rando, N., Verveer, J., Verhoeve, P., et al. 2000, in Proc. SPIE 4008, Optical and IR Telescope Instrumentation and Detectors, ed. M. Iye, \& A. F. Moorwood, 4008, 646

Romani, R. W., Miller, A. J., Cabrera, B., Figueroa-Feliciano, E., \& Nam, S. W. 1999, ApJ, 521, L153

Sargent, W. L. W., Steidel, C. C., \& Boksenberg, A. 1989, ApJS, 69, 703

Zheng, W., Kriss, G. A., Telfer, R. C., Grimes, J. P., \& Davidsen, A. F. 1997, ApJ, 475, 469 